\title{
Composição e distribuição do microfitoplâncton do rio Guamá no trecho entre Belém e São Miguel do Guamá, Pará, Brasil
}

\author{
Composition and distribution of the microphytoplankton from Guamá \\ River between Belém and São Miguel do Guamá, Pará, Brazil
}

\author{
Milena Daisy Ribeiro Monteiro \\ Nuno Filipe Alves Correia de Melo" \\ Marcelo Augusto Moreno da Silva Alves ${ }^{\text {III }}$ \\ Rosildo Santos Paiva ${ }^{\mathrm{N}}$
}

Resumo: As microalgas são responsáveis por grande parte da produtividade primária dos ecossistemas aquáticos. Qualquer distúrbio ambiental que afete a qualidade da água se refletirá na estrutura da comunidade fitoplanctônica e, consequentemente, em toda a teia trófica. Este trabalho apresenta a composição e densidade do microfitoplâncton do rio Guamá, determinadas a partir da análise de 25 amostras coletadas ao longo do trecho compreendido entre Belém e São Miguel do Guamá. Foram identificados 85 táxons pertencentes a Dinophyta (1\%), Cyanophyta (4\%), Chlorophyta (20\%) e Bacillariophyta (75\%). Aulacoseira granulata (Ehrenberg) Simonsen, Coscinodiscus oculus-iridis Ehrenberg, Oscillatoria sp., Polymyxus coronalis L.W. Bail, Surirella guatimalensis Ehrenberg, Thalassiosira eccentrica (Ehrenberg) Cleve e Triceratium favus (Ehrenberg) Wallich foram as espécies mais frequentes. A densidade máxima ficou em 742.000 organismos/litro, verificada na estação 21, e a mínima foi de 9.280 organismos/litro, observada na estação 10.

Palavras-chave: Fitoplâncton. Rio Guamá. Estuário amazônico.

Abstract: The microalgae are responsible for a great part of the primary productivity of the aquatic ecosystems. Any environmental disturbance that affect the quality of water will result in an alteration of the structure of the phytoplankton community and, consequently, of all the trophic web. This work presents the composition and density of microphytoplankton of the Guamá river based on the analysis of 25 samples collected along the passage situated between Belém and São Miguel do Guamá. Were identified 85 taxa belonging to the Dinophyta (1\%), Cyanophyta (4\%), Chlorophyta (20\%), and Bacillariophyta (75\%). Aulacoseira granulata (Ehrenberg) Simonsen, Coscinodiscus oculus-iridis Ehrenberg, Oscillatoria sp. Polymyxus coronalis L.W. Bail, Surirella guatimalensis Ehrenberg, Thalassiorira eccentrica (Ehrenberg) Cleve, and Triceratium favus (Ehrenberg) Wallich were the most frequent species. The maximum density was 742.000 organisms/iter, verified in station 21, and the minimum density observed was 9.280 organisms/iter, found in station 10.

Keywords: Phytoplankton. Guamá River. Amazon estuary.

l Universidade Federal do Pará. Belém, Pará, Brasil (milenadrm@yahoo.com.br).

"Iniversidade Federal Rural da Amazônia. Instituto Sócio-Ambiental e dos Recursos Hídricos. Belém, Pará, Brasil (nunomelo@ufpa.br).

III Universidade Federal Rural da Amazônia. Instituto Sócio-Ambiental e dos Recursos Hídricos. Belém, Pará, Brasil (marcelo.moreno@ufra.edu.br).

vv Universidade Federal do Pará. Instituto de Ciências Biológicas. Belém, Pará, Brasil (rpaiva@ufpa.br).

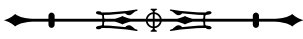




\section{INTRODUÇÃO}

Apesar da quantidade relativamente pequena de água doce de lagos e rios, que somam apenas cerca de 200.000 $\mathrm{km}^{3}$, esse elemento é essencial ao abastecimento para consumo humano, ao desenvolvimento de atividades industriais e agrícolas, e de importância vital aos ecossistemas (Rebouças, 2002). Nestes ecossistemas, a água está relacionada principalmente à manutenção dos organismos responsáveis pela produção primária, os quais servirão de alimentos para os demais elos da teia trófica, permitindo o seu completo funcionamento (Losada et al., 2003; Silva et al., 2005; Ré, 2005).

A comunidade fitoplanctônica de rios, lagos e lagoas está recebendo cada vez mais pesquisas científicas, destacando-se as regiões Norte e Nordeste do país, onde essa comunidade caracteriza-se pela presença de praticamente todos os grupos taxonomicamente importantes (Wetzel, 1993), cujos principais são Cyanophyta, Chlorophyta, Euglenophyta, Bacillariophyta e Dinophyta.

Devido à sensibilidade dos organismos fitoplanctônicos às mudanças ambientais, torna-se claramente necessário conservar os recursos naturais e compreender as implicações do manejo desses recursos (Paiva et al., 2004). Assim, o estudo sobre a comunidade existente no rio Guamá vem corroborar o valor desta ideia, haja vista que objetiva realizar, por meio do conhecimento sobre a área, um levantamento de informações significativas que deem suporte às análises do funcionamento deste ecossistema, assim como de seu estado trófico.

No Pará, dada a sua dimensão geográfica, ainda existem poucos estudos sobre composição fitoplanctônica. O trabalho pioneiro no estado foi realizado por MoreiraFilho et al. (1974), na região da foz do rio Guamá, apresentando o primeiro levantamento sobre a composição do microfitoplâncton deste rio. Neste, foram verificados 24 gêneros, 46 espécies e uma variedade de diatomáceas.

Ainda na região da baía do Guajará e foz do rio Guamá, Paiva \& Leça (1991) observaram concentrações de clorofila variando entre 1,5 e $23 \mu \mathrm{g} /$.
Os trabalhos mais recentes que enfocam a região do rio Guamá são de Paiva et al. (2004), sobre a composição e biomassa primária fitoplanctônica da foz do igarapé Tucunduba, um importante afluente da margem direita da foz do rio Guamá, e o de Paiva et al. (2006), que apresentam considerações ecológicas sobre populações fitoplanctônicas da baía do Guajará e foz do rio Guamá. Os últimos autores identificaram 116 espécies distribuídas entre cianofícea, clorofícea e diatomácea, sendo as diatomáceas o grupo predominante.

Com o objetivo de contribuir para um maior conhecimento sobre o fitoplâncton da região amazônica, o presente estudo apresenta novas informações a respeito da composição, abundância relativa, frequência relativa, densidade e distribuição da microficoflora do rio Guamá (Pará, Brasil).

\section{MATERIAL E MÉTODOS}

\section{LOCALIZAÇÃO E DESCRIÇÃO DA ÁREA}

No presente estudo, foram coletadas 25 amostras de fitoplâncton no trecho compreendido entre os municípios de Belém (1 27' 21" S e 48 30' 14" W) e São Miguel do Guamá (10 37' 37" S e 47० 28' 58” W), Figura 1, no período de 5 a 11 de dezembro de 2005, início do período chuvoso na região.

O rio Guamá mede entre 1.360 e 2.000 m de largura (Ramos, 2004), é afluente do rio Pará e tem 700 km de extensão. Este rio nasce na serra dos Coroados, correndo na direção sul-norte até a cidade de Ourém, situada em sua margem direita, e segue para oeste, onde se encontra com o rio Capim. É navegável numa extensão aproximada de $160 \mathrm{~km}$ até a localidade de São Miguel do Guamá, Pará, onde ocorre um travessão rochoso, e é cruzado pela BR-010. Este rio sofre influência das marés oceânicas em sua foz, recebendo constantes aportes de sedimentos da baía do Guajará, podendo aquele chegar a tornar-se ligeiramente salobro no ápice do período menos chuvoso.

A precipitação da região apresenta um comportamento típico de áreas tropicais de alta pluviosidade, com uma 


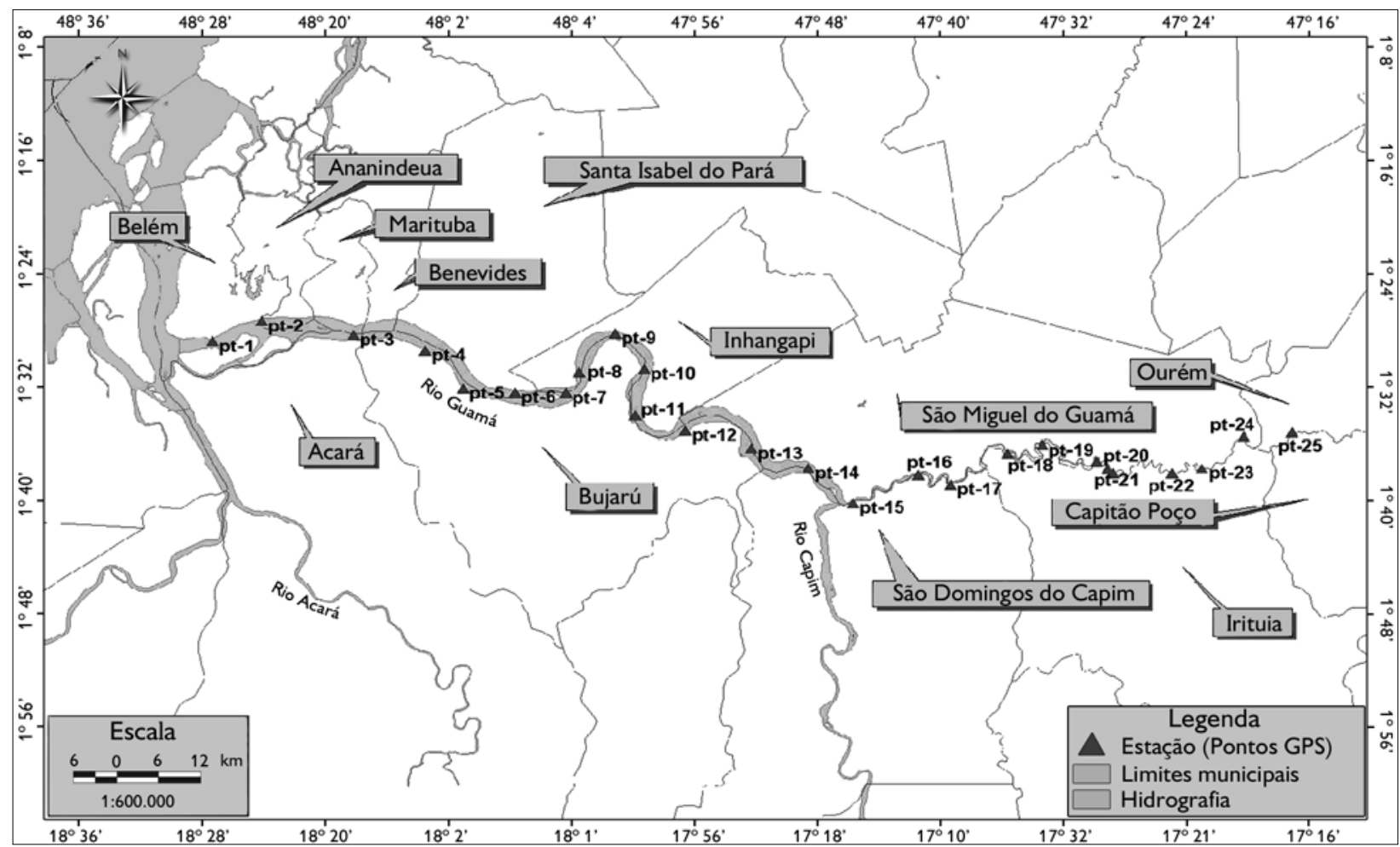

Figura 1. Localização do rio Guamá com a distribuição dos pontos amostrais.

estação chuvosa correspondendo aos meses de dezembro a maio e uma estação seca ou menos chuvosa ocorrendo nos meses de junho a novembro; o mês de maior precipitação é março, seguido de abril, e o de menor precipitação é o mês de novembro, seguido de outubro (Nechet, 1993).

\section{COMPOSIÇÃO FITOPLANCTÔNICA}

As amostras destinadas ao levantamento florístico foram obtidas a partir de arrastos sub-superficias com rede de plâncton de $64 \mu \mathrm{m}$ de abertura de malha, durante três minutos. Após coletadas, as amostras foram acondicionadas em frascos de polietileno e fixadas com formol neutro até a concentração final de 4\%. A composição do fitoplâncton foi determinada utilizando-se lâminas não permanentes, observadas em um microscópio óptico binocular. $\mathrm{Na}$ identificação dos táxons, foram utilizados os trabalhos de Bicudo (1970); Bourrelly (1972); Cleve-Euler (1953);
Desikachary (1959); Förster (1974); Hino \& Tundisi (1984); Hustedt (1930) e Peragallo \& Peragallo (1897-1908). O sistema de classificação utilizado para as diatomáceas foi o de Round et al. (1990); para as cianofíceas, foi o de Desikachary (1959); para as clorofíceas, o de Bourrelly (1972); e para os dinoflagelados, o de Balech (1988).

\section{ABUNDÂNCIA RELATIVA}

A abundância relativa de cada espécie foi determinada por meio de contagem dos 100 primeiros organismos encontrados em lâmina não permanente de cada amostra, seguindo o critério descrito por Lobo \& Leighton (1986).

As espécies que não foram observadas entre os 100 primeiros organismos contados, mas que estiveram presentes na amostra, foram consideradas Raras (R). Para as espécies coloniais, cada colônia foi considerada como sendo um indivíduo, independente do número de células presentes. 


\section{FREQUÊNCIA DE OCORRÊNCIA}

A frequência de ocorrência foi determinada por meio de uma adaptação do cálculo utilizado por Mateucci \& Colma (1982).

\section{DENSIDADE FITOPLANCTÔNICA}

As amostras destinadas à determinação da densidade foram obtidas a partir de coletas de água da superfície e, em seguida, acondicionadas em frascos de polietileno e preservadas com formol neutro até a concentração final de 4\%.

A análise quantitativa foi realizada segundo o método de sedimentação descrito por Uthermöhl (1958). As amostras foram sedimentadas por um período de 24 horas e contadas em microscópio invertido. Para uma melhor visualização das espécies, foi utilizado o corante Rosa de Bengala e os resultados foram expressos em número de indivíduos por litro. Os organismos unicelulares, filamentos, tricomas, colônias e cenóbios foram considerados como um único indivíduo.

\section{RESULTADOS}

\section{COMPOSIÇÃO FITOPLANCTÔNICA}

O microfitoplâncton do rio Guamá se apresentou composto por 82 espécies e três variedades distribuídas entre as divisões Dinophyta, Cyanophyta, Chlorophyta e Bacillariophyta. À Bacillariophyta corresponderam 75\% (64 spp.) do total de espécies identificadas, as quais estão distribuídas em duas classes, 17 ordens, 24 famílias, 29 gêneros e uma variedade. O gênero Coscinodiscus foi o mais representativo, com maior número de espécies (nove), seguido por Nitzschia (cinco) e Actinoptycus, Odontella, Eunotia, Navicula e Surirella, com quatro espécies cada.

A segunda divisão mais abundante qualitativamente foi Chlorophyta. A este grupo pertencem 20\% (17 spp.) do total de espécies, distribuídas em duas classes, quatro ordens, sete famílias, 11 gêneros e duas variedades. Os gêneros com maior representatividade foram Pediastrum e Micrasterias, ambos com quatro espécies.

Cyanophyta foi responsável por 4\% (3 spp.) do total de espécies, distribuída em uma classe, duas ordens, três famílias e três gêneros. Os gêneros presentes foram Oscillatoria, Microcystis e Anabaena, com uma espécie cada.

A divisão Dinophyta foi a que apresentou menor ocorrência, correspondendo a $1 \%$ do total de espécies observadas (Tabela 1).

O maior número de espécies (35) foi registrado na estação 1 e o menor (15) na estação 2. A Figura 2 mostra a distribuição do número de espécies por estação ao longo do rio Guamá, merecendo destaque o trecho compreendido entre as estações 10 e 15, por apresentar o menor número médio de ocorrências, 20 espécies.

A Figura 3 apresenta a composição percentual do microfitoplâncton do rio Guamá por ponto de amostragem e revela a dominância das espécies pertencentes à divisão Bacillariophyta, compondo sempre mais de $60 \%$ do microfitoplâncton em cada ponto de amostragem.

\section{ABUNDÂNCIA RELATIVA}

A categoria Rara se destacou por apresentar o maior número de espécies (32). A categoria dominante foi composta, na sua maioria, por representantes de Bacillariophyta, seguida de Cyanophyta. Das quatro espécies da categoria dominante, três foram diatomáceas, Aulacoseira granulata, Polymyxus coronalis e Surirella guatimalensis, e uma cianofícea, Oscillatoria sp. (Tabela 2).

As análises de abundância mostram Bacillariophyta como o grupo mais representativo ao longo do rio Guamá, seguido pela Cyanophyta e Chlorophyta. Nas estações de 1 a 6, localizadas na foz do rio, as diatomáceas compõem entre 85 e 100\% da abundância.

\section{FREQUÊNCIA DE OCORRÊNCIA}

A Tabela 3 mostra que a categoria que se destaca por conter maior número de táxons é a Esporádica, onde foram registrados 51 do total de 85 que compõem o microfitoplâncton do rio Guamá.

A categoria de maior importância foi a Muito Frequentes, pois nela foram registradas as espécies de maior distribuição ao longo do rio Guamá. A categoria

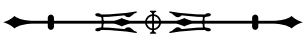


Tabela 1. Composição taxonômica do fitoplâncton do rio Guamá em dezembro de 2005.

Continua

\begin{tabular}{|c|c|}
\hline Táxon & Estações de ocorrência \\
\hline \multicolumn{2}{|l|}{ Cyanophyta } \\
\hline \multicolumn{2}{|l|}{ Cyanophyceae } \\
\hline \multicolumn{2}{|l|}{ Nostocales } \\
\hline \multicolumn{2}{|l|}{ Nostocaceae } \\
\hline Anabaena sp. & $1,3,5,6,7$ \\
\hline \multicolumn{2}{|l|}{ Oscillatoriaceae } \\
\hline Oscillatoria sp. & $1,4,6,7,8,9-24$ \\
\hline \multicolumn{2}{|l|}{ Chroococcales } \\
\hline \multicolumn{2}{|l|}{ Chroococcaceae } \\
\hline Microcystis sp. & $1,2,7-13,15,16,17$ \\
\hline \multicolumn{2}{|l|}{ Dinophyta } \\
\hline \multicolumn{2}{|l|}{ Dinophyceae } \\
\hline \multicolumn{2}{|l|}{ Peridiniales } \\
\hline \multicolumn{2}{|l|}{ Ceratiaceae } \\
\hline $\begin{array}{l}\text { Ceratium fusus } \\
\text { (Ehrenberg) Dujardin }\end{array}$ & $17,18,19,23,24$ \\
\hline \multicolumn{2}{|l|}{ Bacillariophyta } \\
\hline \multicolumn{2}{|l|}{ Coscinodiscophyceae } \\
\hline \multicolumn{2}{|l|}{ Thalassiosirales } \\
\hline \multicolumn{2}{|l|}{ Thalassiosiraceae } \\
\hline $\begin{array}{l}\text { Thalassiosira eccentrica } \\
\text { (Ehrenberg) Cleve }\end{array}$ & $1-22,24,25$ \\
\hline \multicolumn{2}{|l|}{ Skeletonemataceae } \\
\hline $\begin{array}{l}\text { Skeletonema costatum } \\
\text { (Greville) Cleve }\end{array}$ & $1,2,3,23$ \\
\hline \multicolumn{2}{|l|}{ Stephanodiscaceae } \\
\hline $\begin{array}{l}\text { Cyclotella striata } \\
\text { (Kützing) Grunow }\end{array}$ & $2,4-21,25$ \\
\hline \multicolumn{2}{|l|}{ Aulacoseirales } \\
\hline \multicolumn{2}{|l|}{ Aulacoseiraceae } \\
\hline $\begin{array}{l}\text { Aulacoseira granulata } \\
\text { (Ehrenberg) Simonsen }\end{array}$ & $1-21,23,24,25$ \\
\hline \multicolumn{2}{|l|}{ Melosirales } \\
\hline \multicolumn{2}{|l|}{ Melosiraceae } \\
\hline Melosira arctica (Ehrenberg) Dickie & 17 \\
\hline M. islandica O.F. Müller & 17 \\
\hline
\end{tabular}

\section{Coscinodiscales}

Coscinodiscaceae

Coscinodiscus apiculatus Ehrenberg

C. centralis Ehrenberg

C. concinnus W. Smith

C. lineatus Ehrenberg

C. jonesianus (Greville) Ostenfeld

C. oculus-iridis Ehrenberg

C. perforatus Ehrenberg

C. radiatus Ehrenberg

C. rothii (Ehrenberg) Grunow

Eupodiscus antiguos (Cox) Hanna

Actinoptychus splendens

(Shadbolt) Ralfs

Polymyxus coronalis L.W. Bail

Hemidiscaceae

Actinocyclus sp.

Helipeltaceae

Actinoptychus sp.

A. senarius (Ehrenberg) Ehrenberg

A. undulatus (J.W. Bailey) Ralfs

Triceratiales

Triceratiaceae

Cerataulus smithii (Roper) Ralfs

Odontella longicruris (Greville) Hoban

O. mobiliensis (J.W. Bailey) Grunow

O. regia (Ostenfeld) Simonsen

O. sinensis (Greville) Grunow

Triceratium favus (Ehrenberg) Wallich

T. favus var. quadrata Grunow

T. brockeii G. Leuduger-Fortmorel

Biddulphiales

Biddulphiaceae

Terpsinoë musica Ehrenberg

\begin{tabular}{l|c}
\hline $\begin{array}{l}\text { Bellerocheaceae } \\
\text { Bellerochea sp. }\end{array}$ & $16,18,21,22$ \\
\hline Rhizosoleniales & \\
Rhizosoleniaceae & $1-11,14$ \\
Rhizosolenia hebetata J.W. Bailey &
\end{tabular}

$8,16,19,23,24,25$

$1,2,9,10,11,13,15,16,19,22$

$1,2,4-9,13,16,18-22,25$

$15,16,17,20$

8

$1-12,14,16,18-25$

$1-5,7-11,17,19,20,22,24$

$1,2,4,8,12,13,24$

$1-5,7-11,14,16,18,19$

$7,8,18,19,20,23$

16

$1-23$

19

$1,6,16,24$

23

$3-9,12,15-21$

$1,7,9,16,19-21$

$13,14,18$

$1,2,3,6$

$1,2,5,6$

16

$1-12,14,16-21,23,25$

$2,4,20,21$

$1,2,5-9,11,13,14,16,19,20$ 
Tabela 1.

\begin{tabular}{l|c}
\hline \multicolumn{1}{c|}{ Táxon } & Estações de ocorrência \\
\hline Chaetocerales & \\
Chaetoceraceae & \\
Bacteriastrum delicatulum Cleve & $9,10,12$ \\
B. hyalinum Lauder & $10,11,12$ \\
\hline Leptocylindrales & \\
Leptocylindraceae & 1,2 \\
Leptocylindrus danicus Cleve & $1,2,3$ \\
L. minimus Grunow & \\
\hline Fragilariophyceae & \\
Fragilariales & \\
Fragillariaceae &
\end{tabular}

Fragilaria hyalina (Kützing) Grunow ex Van Heurck

$20,23,24,25$

F. striatula Lyngbye

\begin{tabular}{|c|c|}
\hline \multicolumn{2}{|l|}{ Raphoneidales } \\
\hline Raphoneidaceae & \\
\hline Raphoneis nitida (Gregory) Grunow & 24 \\
\hline \multicolumn{2}{|l|}{ Licmophorales } \\
\hline \multicolumn{2}{|l|}{ Licmophoraceae } \\
\hline Licmophora sp. & 19 \\
\hline L. ovata (W. Smith) Grunow & 19 \\
\hline \multicolumn{2}{|l|}{ Thalassionematales } \\
\hline \multicolumn{2}{|l|}{ Thalassionemataceae } \\
\hline $\begin{array}{l}\text { Thallassionema frauenfeldii } \\
\text { (Grunow) Hallegraeff }\end{array}$ & 25 \\
\hline $\begin{array}{l}\text { T. nitzschioides (Grunow) } \\
\text { Mereschhkowsky }\end{array}$ & 24,25 \\
\hline \multicolumn{2}{|l|}{ Eunotiales } \\
\hline \multicolumn{2}{|l|}{ Eunotiaceae } \\
\hline Eunotia sp. & $11,16,19,23,25$ \\
\hline E. gibbosa Grunow in Van Heurck & 22,25 \\
\hline E. femoriforme (Patrick) Hustedt & 22 \\
\hline E. flexuosa (Brébisson) Kützing & $15,20,22,23$ \\
\hline \multicolumn{2}{|l|}{ Naviculaceae } \\
\hline Navicula sp. & $2,3,8,14,16,19,20,23,25$ \\
\hline N. elegans W. Smith & 19 \\
\hline N. viridula (Kützing) Kützing & $1,4,9-11,13,14,16,18,19,21-24$ \\
\hline
\end{tabular}

\begin{tabular}{l|l}
\hline $\begin{array}{l}\text { Pleurosigmataceae } \\
\text { Pleurosigma sp. }\end{array}$ & $1,2,5,8,16,21$ \\
\hline Thalassiosiphysales & \\
Catenulaceae &
\end{tabular}

$8,11,14$

Entomoneis alata Ehrenber

Bacillariales

Bacillariaceae

Bacillaria paxillifera (Müller) Hendey

1, 2, 3

Nitzschia sp.

N. longissima (Brébisson) Ralfs

N. distans Gregory

N. closterium (Ehrenberg) W. Smith

$2,7,15,18,20-25$

N. seriata Cleve

Surirellales

Surirellaceae

Surirella brebissoni Kramer

\& Lange-Bertalot

S. guatimalensis Ehrenberg

7, 11,22

S. robusta Ehrenberg

S. splendida (Ehrenberg) Kützing

$1-19,22,23$

1, 2, 4-10, 13-15, 18, 22

Chlorophyta

Euchlorophyceae

Chlorococcales

Hydrodictyaceae

Pediastrum duplex Meyen

P. duplex var. duplex Meyen

$2,13,23$

P. duplex var. punctatum (Krieger) Parra

13

8,9

P. simplex (Meyen) Lemmermann $\quad 6$

Scenedesmaceae

Scenedesmus sp.

$1,2,3,11,12,15$

Tetrasporales

Palmellaceae

Sphaerocystis sp.

$1,2,11,25$

Zygnematophyceae

Desmidiales

Closteriaceae

Closterium sp.

$18,20,22-25$

Cosmarium sp.

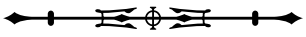


Tabela 1.

Conclusão

\begin{tabular}{l|c}
\hline \multicolumn{1}{c|}{ Táxon } & Estações de ocorrência \\
\hline Desmidiaceae & 12,22 \\
Spondylosium sp. & $1,5,7-14$ \\
Staurastrum pseudosebaldi Wille & 16,17 \\
Bambusina sp. & $1,8,13,16,22-24$ \\
Micrasterias sp. & $21,22,25$ \\
M. mahabuleshwarensis J. Hobson & $21,23,24$ \\
M. radiosa Ralfs & 20 \\
M. schweinfurthii Cohn & \\
Zygnematales & $1,9,13,17,19-25$ \\
Peniaceae & \\
Gonatozygon kinahani & \\
(W. Archer) Rabenhost & $1,2,6-9,14-16,20-25$ \\
\hline Zygnemataceae & \\
Spirogyra sp. &
\end{tabular}

apresentou um total de sete táxons pertencentes à divisão Bacillariophyta: Aulacoseira granulata, Coscinodiscus oculus iridis, Polymyxus coronalis, Surirella guatimalensis, Thalassiosira eccentrica e Triceratium favus, sendo que Thalassiosira eccentrica e Aulacoseira granulata foram as de maior frequencia, presentes em 24 das 25 amostras coletadas ao longo do rio.

\section{DENSIDADE DO MICROFITOPLÂNCTON}

A densidade do microfitoplâncton no rio Guamá variou entre 9.280 org/itro (estação 21) e 742.000 org/litro (estação 10), sendo os maiores valores observados no trecho mais sinuoso do rio, entre os pontos de coleta $8 \mathrm{e}$ 14, e os menores na parte mais interna, entre os pontos 17 e 22 (Figura 4).

\section{DISCUSSÃO E CONCLUSÃO}

O microfitoplâncton representa parte importante dos produtores primários aquáticos. É composto por uma comunidade heterogênea de microorganismos fotossinteticamente ativos, com necessidades fisiológicas iguais aos demais organismos clorofilados, isto é, requerem condições físicas e químicas adequadas para crescer e reproduzir-se.

O microfitoplâncton do rio Guamá se apresentou composto por diatomáceas, clorofíceas e cianofíceas. A dominância de representantes de Bacillariophyta na ficoflora do rio Guamá foi evidente (75\%). Resultados semelhantes foram anteriormente verificados para a região, pois nos trabalhos de Moreira-Filho et al. (1974) e de Paiva et al. (2006), ambos com amostragens realizadas na foz do rio Guamá, as diatomáceas predominaram sobre os demais grupos. Paiva et al. (2004), em seu estudo sobre a composição e biomassa fitoplanctônica na foz do igarapé Tucunduba, também constataram a dominância das Bacillariophyta, isto, segundo os autores, devido à forte influência exercida pelo rio Guamá sobre o igarapé, a qual se intensifica no período da preamar.

Em outro trabalho de Paiva et al. (2006), sobre a composição específica do microfitoplâncton da baía do Guajará, foi verificado que as diatomáceas formaram o grupo de maior representação, com abundância em torno de $56,2 \%$.

Segundo Riley (1967) e Smayda (1983), as diatomáceas se destacam como o principal grupo de organismos componentes do microfitoplâncton estuarino, inferindo, portanto, sobre o caráter ecológico da região objeto deste estudo. No entanto, a sua distribuição é mais abrangente. Segundo Reviers (2006), as diatomáceas estão amplamente distribuídas na natureza e estão aptas a colonizar todos os meios aquáticos. Isso leva à discussão sobre sua presença e caráter indicador de ambiente, haja vista que, segundo trabalhos de Gessner \& Simonsen (1967), é importante atentar para o fato de as valvas de diatomáceas serem resistentes, podendo ser carregadas através dos rios e mascarar a composição florística do ambiente.

A segunda divisão com maior riqueza de espécies, Chlorophyta, compõe o microfitoplâncton predominantemente de ambientes limnéticos e é 


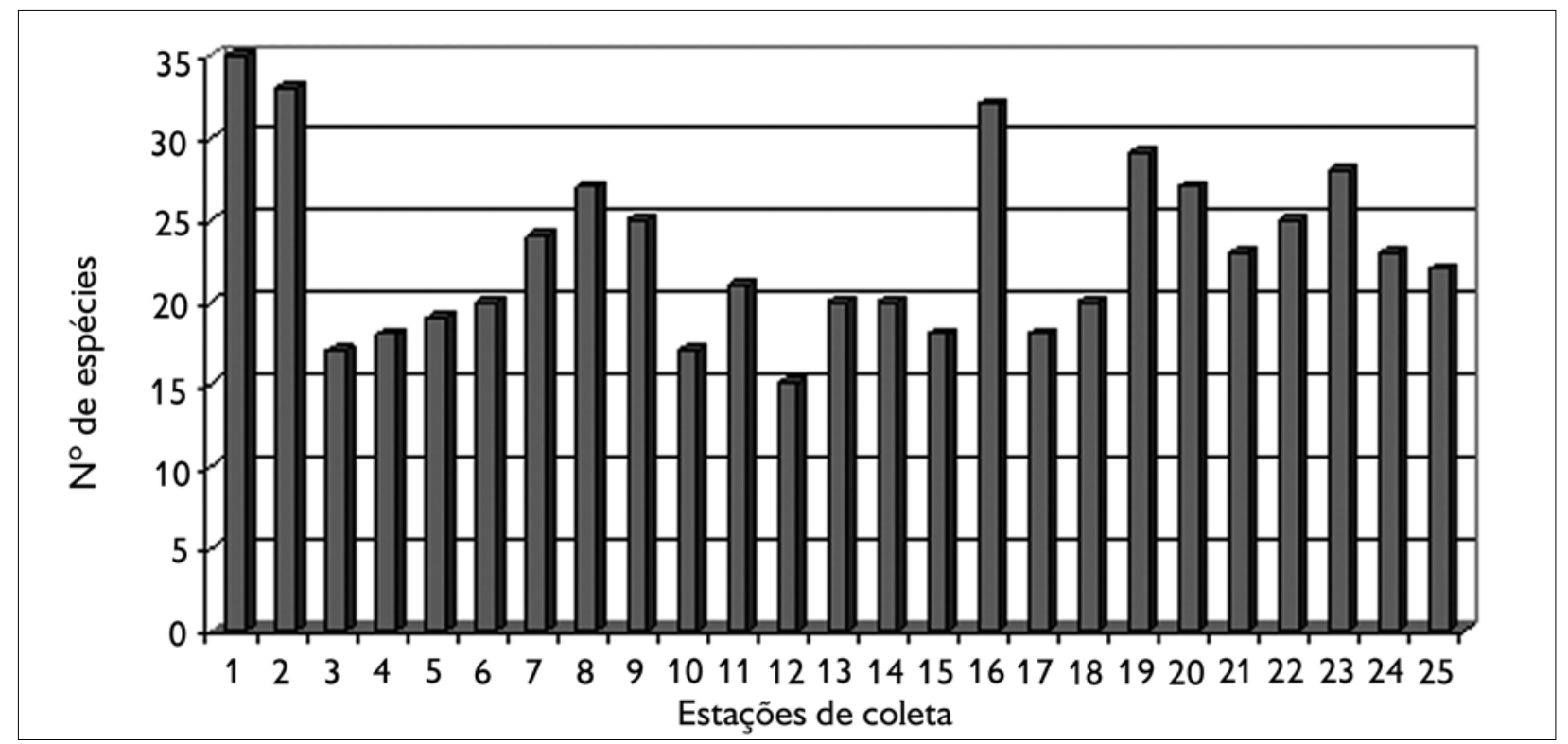

Figura 2. Distribuição do número total de táxons por ponto de coleta no rio Guamá em dezembro de 2005.

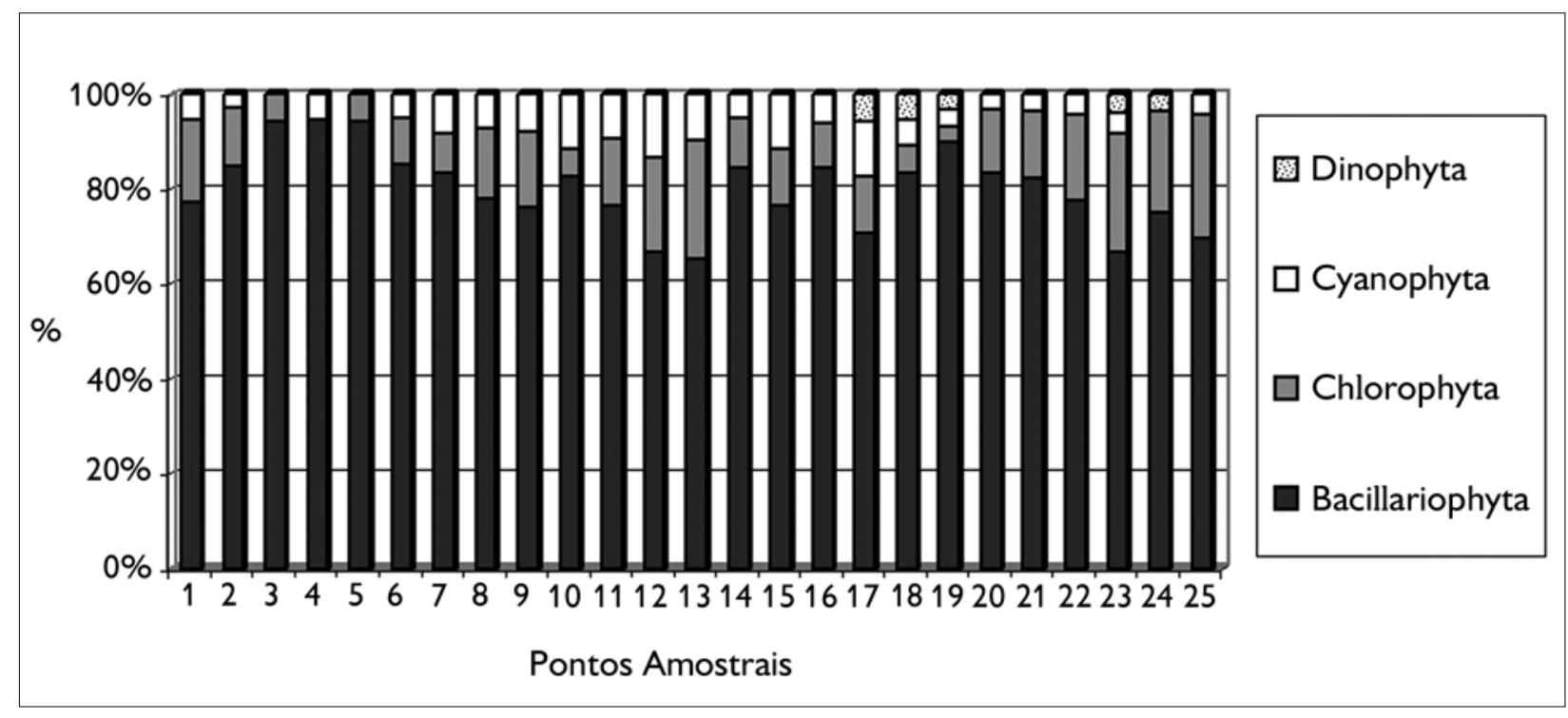

Figura 3. Composição percentual do microfitoplâncton por ponto de coleta no rio Guamá em dezembro de 2005.

encontrada em quase todos os ambientes (Reviers, 2006). As clorofíceas também foram observadas em trabalhos anteriores realizados na região da baía do Guajará e rio Guamá, como, por exemplo, o de Paiva et al. (2006).

A representatividade de Cyanophyta foi de apenas $3 \%$. As cianofíceas, terceiro grupo em termos de ocorrência na área de estudo, também foram observadas no trabalho de Paiva et al. (2006), os quais verificaram as clorofíceas e cianofíceas presentes em menor proporção em relação às diatomáceas. Dinophyta foi a que apresentou menor representatividade (1\%). Segundo Reviers (2006), este grupo é, sobretudo, marinho, existindo, porém,

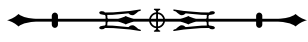


Tabela 2. Abundância relativa do microfitoplâncton do rio Guamá em dezembro de 2005.

\begin{tabular}{c|l}
\hline Categorias & \multicolumn{1}{c}{ Espécies } \\
\hline $\begin{array}{c}\text { Dominante } \geq 70 \% \\
\text { Abundante }<70 \%-40 \%\end{array}$ & $\begin{array}{l}\text { Aulacoseira granulata, Polymyxus coronalis, Surirella guatimalensis e Oscillatoria sp. } \\
\text { Aulacoseira granulata e Polymyxus coronalis } \\
\begin{array}{c}\text { Pouco Abundante } \\
<40 \%-10 \%\end{array} \\
\text { Actinoptychus undulatus, Coscinodiscus perforatus, Coscinodiscus rothii, Cyclotella striata, Staurastrum } \\
\text { pseudosebaldi, Spyrogyra sp., Thalassiosira eccentrica e Gonatozygon kinahani }\end{array}$ \\
& $\begin{array}{l}\text { Nitzschia seriata, Pinnularia stauroptera, Sphaerocystis sp., Triceratium favus var. quadrata, Eunotia sp., Surirella } \\
\text { robusta, Terpsinoë musica, Lepdocylindricus danicus, Thalassionema nitzschioides e Coscinodiscus radiatus }\end{array}$ \\
\hline
\end{tabular}

Tabela 3. Frequência de ocorrência do microfitoplâncton do rio Guamá em dezembro de 2005.

\begin{tabular}{|c|c|}
\hline Categorias & Espécies \\
\hline $\begin{array}{l}\text { Muito Frequentes } \\
\quad \leq 80 \%\end{array}$ & $\begin{array}{l}\text { Aulacoseira granulata, Coscinodiscus oculus iridis, Oscillatoria sp., Polymyxus coronalis, Surirella guatimalensis, } \\
\text { Thalassiosira eccentrica, Triceratium favus }\end{array}$ \\
\hline $\begin{array}{l}\text { Frequentes } \\
<80 \%-50 \%\end{array}$ & $\begin{array}{l}\text { Actinoptycus undulatus, Cyclotella striata, Coscinodiscus concinnus, C. perforatus, C. rothii, Navicula viridula, } \\
\text { Pinnularia stauroptera, Spyrogyra sp., Surirella robusta, Triceratium brokeii }\end{array}$ \\
\hline $\begin{array}{l}\text { Pouco Frequentes } \\
<50 \%-20 \%\end{array}$ & $\begin{array}{l}\text { Bacillaria paxillifera, Cerataulus smithii, Closterium sp., Coscinodiscus apiculatus, C. centralis, C. radiatus, Eupodiscus } \\
\text { antiquos, Gonatozygon kinahani, Micrasterias sp., Microcystis sp., Navicula sp., Nitzschia distans, Nitzschia sp., } \\
\text { Pleurosigma sp., Rhizosolenia hebetata, Scenedesmus sp., Staurastrum pseudosebaldi, Surirella splendida }\end{array}$ \\
\hline $\begin{array}{l}\text { Esporádica } \\
<20 \%\end{array}$ & $\begin{array}{l}\text { Actinocyclus sp., Actinoptychus sp., A. senarius, A. splendens, Anabaena sp., Bacteriastrum delicatum, B. } \\
\text { hyalinum, Bambusina sp., Bellerochea sp., Ceratium fusus, Coscinodiscus lineatus, C. jonesianus, Cosmarium } \\
\text { sp., Entomoneis alata, Eunotia sp., E. indica, E. monodon, E. triadon, Fragilaria hyalina, F. striatula, } \\
\text { Leptocylindrus danicus, L. minimus, Licmophora var. quadrata, Licmophora var. ovata, Mellosira artica, M. } \\
\text { islandica, Micrasterias mahabulesshwarensis, M. radiosa, M. schweinfurthii, Navicula bory, N. elegans, N. } \\
\text { subsalina, Nitzschia closterium, N. longissima, N. seriata, Odontella mobiliensis, O. regia, O. sinensis, O. } \\
\text { longricuris, Pediastrum duplex, P. duplex var. duplex, Pediastrum duplex var. punctatum, P. simples, Rhaponeis } \\
\text { nitida, Sphaerocystis sp., Skeletonema costatum, Spondylosium sp., Surirella brebissoni, Terpsinoë musica, } \\
\text { Thalassionema frauenfeldii, T. nitzschioides, Triceratium favus var. quadrata }\end{array}$ \\
\hline
\end{tabular}

numerosas espécies dulcícolas. Elas são encontradas principalmente no plâncton e sua distribuição dá-se dos pólos aos trópicos (Reviers, 2006).

Em termos de abundância relativa, foi verificada a dominância das diatomáceas, correspondendo a mais de $70 \%$ do total de táxons identificados em cada uma das 25 amostras do rio Guamá. Merece destaque Polymyxus coronalis, a qual é, segundo Paiva et al. (2006), indicadora de águas salobras da região amazônica.

Polymyxus coronalis e Aulacoseira granulata também se destacaram como Muito Frequentes, juntamente com Coscinodiscus oculus iridis, Surirella guatimalensis, Thalassiosira eccentrica e Triceratium favus. Este resultado corrobora o observado por Paiva et al. (2004), onde os autores observaram, na mesma categoria, a predominância de diatomáceas, com especial destaque para as espécies Polymyxus coronalis e Aulacoseira granulata por apresentarem valores elevados de frequência de ocorrência.

A densidade do microfitoplâncton observada, variando de 9.280 a 742.000 org/litro, foi muito superior ao registrado por Cardoso et al. (2006) na mesma área de estudo, em novembro de 2004, cujos valores variaram entre 222 e 244.370 org/litro. No entanto, assim como Cardoso et al. (2006), o presente estudo identificou as estações intermediárias, de 8 a 14, como as que apresentaram os maiores valores

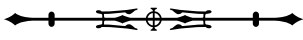




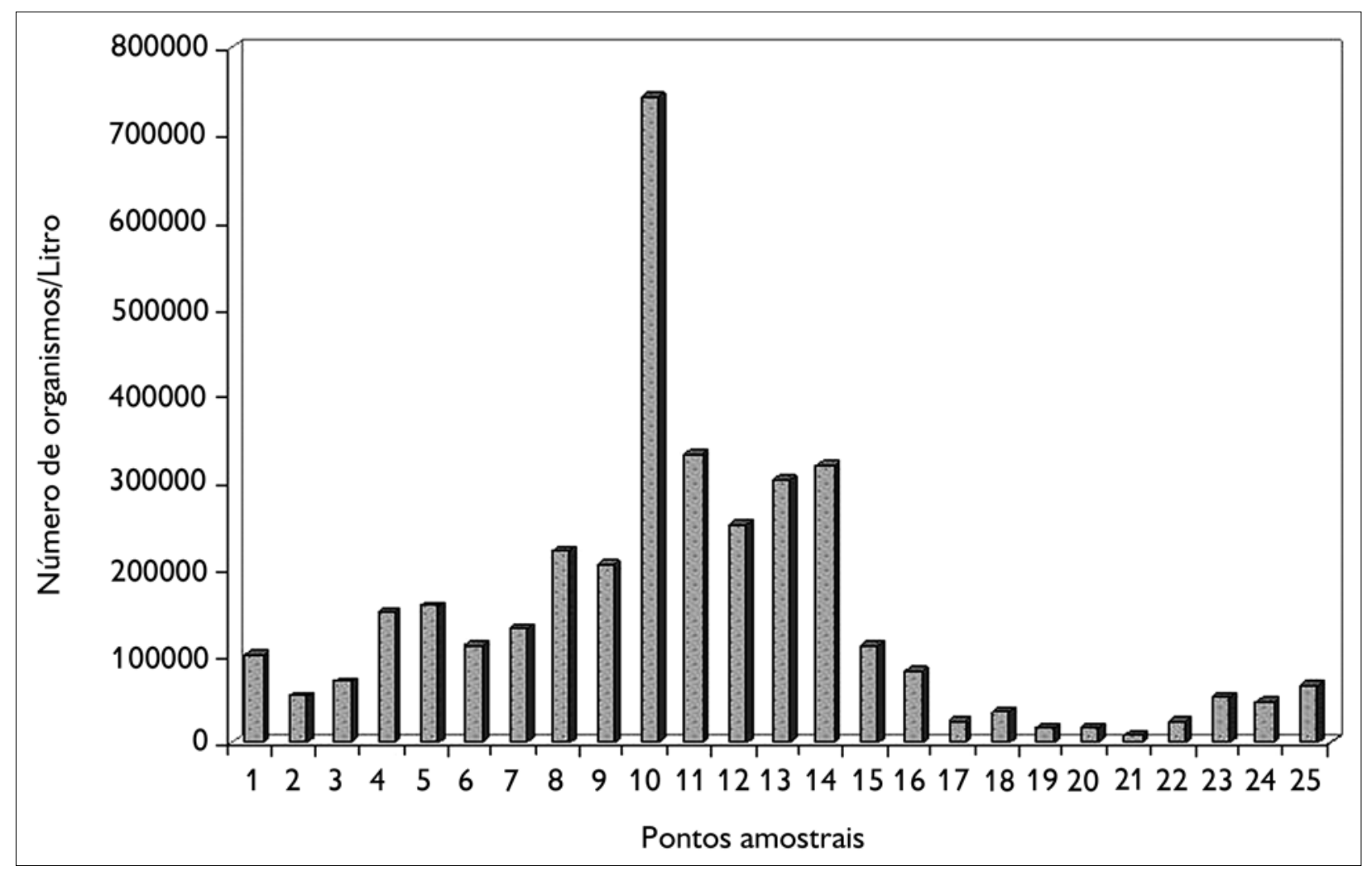

Figura 4. Variação da densidade do microfitoplâncton do rio Guamá em dezembro de 2005.

de densidade. Isso certamente está relacionado ao fato de que estas estações estão localizadas na região mais tortuosa do rio, o que reduz as características de um corpo d'água tipicamente lêntico, favorecendo o florescimento do microfitoplâncton.

O ambiente analisado apresenta uma distribuição da ficoflora bastante heterogênea, de maneira que os grupos menos representativos foram observados nas estações mais internas e com um número maior de espécies. No entanto, as diatomáceas não perdem a posição de dominância. Esta distribuição do microfitoplâncton também possibilita inferir sobre as características ecológicas do trecho do rio Guamá compreendido entre Belém e São Miguel do Guamá, ou seja, de acordo com a composição fitoplanctônica, pode-se identificar o seu grau estuarino ou limnético. Segundo Cardoso et al. (2006), os pontos do rio Guamá mais limnéticos têm uma maior ocorrência de
Chlorophyta, enquanto que a parte estuarina caracterizase pela predominância de diatomáceas. Desta forma, em função da composição do microfitoplâncton apresentada, é possível afirmar que o trecho do rio Guamá que vai de sua desembocadura até, aproximadamente, $70 \mathrm{~km}$ internos tem uma ficoflora estuarina, sendo que esta dimensão, como nos demais rios da região amazônica, poderá variar em função do período de maior ou menor precipitação pluviométrica, assim como em função das marés de sizígia ou quadratura e preamar ou baixa mar.

\section{REFERÊNCIAS}

BALECH, E., 1988. Los dinoflagelados del Atlântico sudoccindetal: 1-310. Instituto Espanõl de Oceanografia (Publicaciones especiales), Madrid.

BICUDO, C. E. M., 1970. Algas de águas continentais brasileiras: chave ilustrada para identificação dos gêneros: 1-228. EDUSP, São Paulo. 
BOURRELLY, P., 1972. Les algues d'eau douce: Initiation à la systématique. Tome I: Les algues vertes: 1-509. Éditions M. Boubée \& Cie. (Collection "Faunes et Flore Actuelly"), Paris.

CARDOSO, F. F., C. O. COSTA, R. S. PAIVA, N. F. A. C. MELO \& M. D. R. MONTEIRO, 2006. Distribuição espacial e variação da densidade do microfitoplâncton no rio Guamá, trecho compreendido entre Belém e São Miguel do Guamá - PA. Anais da III Semana de Ciências Aquáticas 1-10.

CLEVE-EULER, A., 1953. Die Diatomeen von Schweeden und Finland. Bihang K. Svenska Vetenskapsakademiens Handlingar 2(1): 1-163.

DESIKACHARY, T. S., 1959. Cyanophyta: 1-686. Concil of Agricultura Researcer, Indian.

FÖRSTER, V. K., 1974. Amazonische demidieen: 135-242. Amazoniana, Manaus.

GESSNER, F. \& R. SIMONSEN, 1967. Marine Diatoms in the Amazon. Limnology and Oceano 12(4): 709-711.

HINO, K. \&J. TUNDISI, 1984. Atlas de algas da Represa do Broa: 1-143. Universidade Federal de São Carlos, São Carlos.

HUSTEDT, F., 1930. Die Kieselalgen. Deutschlands, Osterreichs und der Schweiz. In: L. RABENHOST (Ed.): Kryptogamen-flora von Deutschlands, Osterreich und der Schweiz: 1-920. Akademische Verlagsgesellschaft, Portigh.

LOBO, E. \& G. LEIGHTON, 1986. Estruturas comunitárias de las fitocenosis planctônicas de los sistemas de desembocaduras de rios y esteros de la zona central de Chile. Revista Biologia Marina 22: 1-29.

LOSADA, A. P. M., F. A. N. FEITOSA \& I. C. LINS, 2003. Variação sazonal e espacial da biomassa fitoplânctonica nos estuários dos rios Ilhetas e Mamucaba (Tamandaré-PE) relacionada com parâmetros hidrológicos. Tropical Oceanography 31(1): 1-29.

MATEUCCI, S. D. \& A. COLMA, 1982. Metodología para el estudio de la vegetación: 1-168. Collecion de Monografias Cientificas (Série Biologia), Washington.

MOREIRA-FILHO, H., I. M. VALENTE-MOREIRA \& I. I. T. CECY, 1974. Diatomáceas do rio Guamá, Foz do rio - Belém - Estado do Pará. Leandra 3/4(4/5): 123-135.

NECHET, D., 1993. Análise da precipitação em Belém-Pará, de 1986 a 1991. Boletim de Geografia Teorítica 23(45/46): 100-156.

PAIVA, R. S. \& E. E. LEÇA, 1991. Variação da densidade (células/ litro) do fitoplâncton na baía do Guajará (Pará-Brasil). Anais do IV Encontro Brasileiro de Plâncton 123-138.
PAIVA, R. S., N. MELO, P. GOUVÊA, C. RAMOS \& S. COSTA, 2004. A ocupação urbana como fonte poluidora dos corpos d'água na região amazônica. Estudo de caso: Composição e biomassa primária da foz do Igarapé Tucunduba (Belém-Pará). In: S. UNLY \& E. L. SOUZA (Eds.): A questão da água na Grande Belém: 231 247. Casa de Estudos Germânicos, Belém.

PAIVA, R. S., E. E. LEÇA, J. Z. O. PASSAVANTE, M. G. G. SILVACUNHA \& N. F. A. C. MELO, 2006. Considerações ecológicas sobre o fitoplâncton da baía do Guajará e foz do rio Guamá (Pará-Brasil). Boletim do Museu Paraense Emílio Goeldi. Ciências Naturais 1(2): 133-146.

PERAGALLO, H. \& M. PERAGALLO, 1897-1908. Diatomees marines de France et des districts maritimes voisins: 1-492. M. J. Tempère, Paris.

RAMOS, J., 2004. Poluição e contaminação da orla de Belém-PA. In: L. UHLY \& E. L. SOUZA (Eds.): A questão da água na Grande Belém: 121-148. Casa de Estudos Germânicos, Belém.

RÉ, P., 2005. Ecologia marinha e estuarina: 1-64. Faculdade de Ciências da Universidade de Lisboa, Lisboa.

REBOUÇAS, A. C., 2002. Água doce no mundo e no Brasil. In: A. C. REBOUÇAS, B. BRAGA \&J. G. TUNDISI (Eds.): Águas doces no Brasil: capital ecológico, uso e conservação: 1-37. Escrituras Editora, São Paulo.

REVIERS, B., 2006. Biologia e filogenia das algas: 1-280. Artmed, Porto Alegre.

RILEY, G. A., 1967. The Plankton of estuaries. In: G. H. LAUFF (Ed.): Estuaries: 316-326. American Association for the Advancement of Science, Washington.

ROUND, F. E., R. M. CRAWFORD \& D. G. MANN, 1990. The Diatoms: Biology and Morphology of the genera: 1-747. Cambridge University Press, Cambridge.

SILVA, M. R., M. G. G. SILVA-CUNHA, F. A. N. FEITOSA \& K. MUNIZ, 2005. Estrutura da comunidade fitoplanctônica na baía de Tamandaré (Pernambuco, Nordeste do Brasil). Tropical Oceanography 33(2): 159-177.

SMAYDA, T. J., 1983. The phytoplankton of estuaries. In: B. H. KETCHUM (Ed.): Estuaries and Enclosed Seas: 65-101. Elsevier Scientific Publ., Amsterdam.

UTHERMÖHL, H., 1958. Zur Vervollkomninung der quantitativen. Phytoplankton - Methodik Mitteilungen. Internationale Vereinigung. Limnologie 9: 1-38.

WETZEL, R. G., 1993. Limnologia: 1-905. Fundação Calouste Gulbenkian, Lisboa.

Recebido: 08/01/2008

Aprovado: 24/08/2009

Responsabilidade editorial: Anna Luiza Ilkiu Borges 
